\title{
Torsión esplénica con localización inusual, reporte de caso
}

\author{
Karen Daniela Manchola Narváez \\ Héctor Conrado Jiménez Sánchez** \\ Keila Mendoza Borja** \\ Edgar Forero ${ }^{* * * *}$
}

\begin{abstract}
*Especialista en Epidemiología, Residente Pediatría Il año Universidad Surcolombiana, Hospital Hernando Moncaleano. Neiva, Huila, Colombia. **Cirujano General, Especialista en Epidemiologia clínica, Universidad Surcolombiana, Fellow de Cirugía Vascular Universidad del Bosque ***Especialista en Epidemiología, Residente Pediatría III año Universidad Surcolombiana. Hospital Hernando Moncaleano. Neiva, Huila, Colombia. ****Cirujano pediatra Universidad Nacional de Colombia. Hospital Hernando Moncaleano. Neiva, Huila, Colombia.

Correspondencia: Dr. Héctor Conrado Jiménez Sánchez. Correo electrónico: heconjisan@gmail.com
\end{abstract}

\section{Resumen}

El bazo errante es una entidad clínica muy poco frecuente en niños, representando menos del $0.25 \%$ de todas las esplenectomías. Puede ocurrir por la ausencia congénita de ligamentos suspensorios del bazo, o debido a una laxitud adquirida de los mismos. El espectro clínico va desde condición asintomática con hallazgo incidental, hasta presentación de abdomen agudo. El conocimiento de esta condición y de sus hallazgos radiológicos, juegan un papel esencial para lograr un diagnóstico correcto y tratamiento quirúrgico oportuno. La preservación del bazo más esplenopexia es el procedimiento de elección. Se presenta un paciente de 4 años con antecedente de cirugía antirreflujo, en quien se encontró bazo ectópico errante con torsión esplénica, que fue manejado exitosamente con conservación del bazo y esplenopexia. MÉD.UIS.2021;34(1): 113-18

Palabras clave: Ectopia del bazo. Torsión aguda. Esplenoptosis en pediatría. Esplenopexia.

\section{Splenic torsion with unusual location, case report}

\section{Abstract}

Wandering spleen is an uncommon clinical entity in children, representing less than $0,25 \%$ of all splenectomies. It is caused by laxity or absence of the supporting splenic ligaments. Its clinical spectrum varies from asymptomatic disease with incidental finding to acute abdominal pain due to torsion. The knowledge of this condition and its radiological findings plays a crucial role in reaching the correct diagnosis and timely surgical treatment. Spleen preservation with splenopexy is recognized as the procedure of choice. We report a case of a four year old patient with a history of anti-reflux surgery, with a wandering spleen and splenic torsion, successfully managed with spleen conservation and splenopexy. MÉD.UIS.2021;34(1): 113-18

Keywords: Wandering spleen. Acute Torsion. Pediatric Splenoptosis. Splenopexy.

¿Cómo citar este artículo?: Manchola KD, Jiménez HC, Mendoza K, Forero E. Torsión esplénica con localización inusual, reporte de caso. MÉD.UIS.2021;34(1):113-18. doi: 10.18273/revmed.v34n12021012 


\section{Introducción}

El bazo ectópico (BE), también conocido como errante $O$ esplenoptosis, es una patología infrecuente 1 . La primera descripción detallada de esta entidad clínica fue hecha por Van Horne en 1667 como hallazgo incidental en una autopsia ${ }^{2}$. La verdadera incidencia del bazo errante es incierta, pero representa menos del $0.2 \%$ de las esplenectomías 3 . Se observa comúnmente en niños donde parece haber un predominio masculino en el primer año de vida (2.5: 1) y en mujeres durante la tercera década de la $\mathrm{vida}^{4}$, especialmente en edad reproductiva. Es una condición potencialmente mortal si ocurre torsión aguda junto con infarto esplénico ${ }^{3}$.

Puede ocurrir por la ausencia congénita, mal desarrollo de los ligamentos suspensorios del bazo, o debido a una laxitud adquirida de los mismos, permitiéndole una movilidad excesiva que hace que el bazo "deambule" desde su posición habitual, en el cuadrante superior izquierdo", a localizaciones ectópicas en la cavidad abdominal ${ }^{6}$.

La forma congénita es el resultado de la falta de desarrollo de las uniones ligamentosas primarias del bazo. Se han descrito también diversas anomalías adquiridas que pueden causar laxitud de los ligamentos de soporte, como lo son los cambios hormonales descritos en mujeres en edad reproductiva y con embarazos múltiples, lo que explicaría la presentación predominante en mujeres en pacientes adultos ${ }^{4}$. Tanto las afecciones congénitas como las adquiridas provocan un pedículo alargado, que está predispuesto a la torsión y puede provocar un infarto parcial o completo debido a que los vasos esplénicos cursan dentro de él?.

El espectro clínico va desde condición asintomática con hallazgo incidental, hasta manifestación de abdomen agudo. Las presentaciones clínicas más frecuentes en niños son el dolor abdominal agudo debido a la torsión, seguido de sensación de masa abdominal ${ }^{4,5}$. Lo variado e inespecífico de su presentación clínica puede generar confusiones y un diagnóstico tardío con predisposición a una torsión hilar e infarto posterior ${ }^{6}$, lo que lo convierte en una emergencia abdominal potencialmente mortal?.

Debido a la dificultad en el diagnóstico clínico, las pruebas de imagen juegan un papel esencial para su detección oportuna. La tomografía computarizada con doble contraste (TACC) es el estudio de imagen de elección para el diagnóstico de bazo ectópico cuando se sospecha torsión. Los hallazgos de la TACc incluyen la posición ectópica del bazo, la grasa circundante en el hilio esplénico, que puede estar acompañada o no de torsión de la cola pancreática, y la apariencia en espiral del pedículo esplénico o "whirl sign", hallazgo patognomónico de torsión esplénica ${ }^{7,8,9,10}$.

El tratamiento del bazo errante es quirúrgico. La decisión de realizar esplenopexia vs espenectomía, depende del éxito de la detorsión y de la viabilidad del bazo (cuando hay torsión, la esplenectomía debe realizarse en condición de infarto esplénico masivo y/o trombosis venosa esplénica) ${ }^{5}$.

Se presenta el caso de un paciente en edad preescolar, que presentó dolor abdominal asociado a sensación de masa en hipogastrio, posteriormente se confirmó la presencia de bazo ectópico con torsión del pedículo vascular, en el cual se llevó a cabo detorsión exitosa con esplenopexia.

Se describe el siguiente caso con el objetivo de resaltar la importancia del conocimiento de esta entidad que, aunque es poco frecuente, puede dar lugar a complicaciones como la torsión del pedículo esplénico, que pueden convertirse en emergencia. Es necesario lograr la confirmación del diagnóstico y tratamiento oportunos, para impactar de manera positiva en la salud del paciente.

\section{Caso clínico}

Paciente masculino de 4 años de edad procedente de Guamo (Tolima), con antecedente de cirugía antirreflujo a los 2 años de vida (funduplicatura Nissen (FN) + piloromiotomia anterior) y dolor abdominal crónico estudiado 12 meses previo al ingreso actual, donde se realizó TACc abdominal que evidenció leve esplenomegalia, se descartaron patologías infecciosas o alteraciones hematológicas (Ver Figura 1).

Consultó al servicio de urgencias en compañía de la madre por cuadro clínico de una semana de evolución, consistente en dolor abdominal intermitente, agudizado cuatro días previo a su ingreso, asociado a síntomas respiratorios altos, picos febriles cuantificados $\left(38.5^{\circ} \mathrm{C}\right)$ que cedían con la administración de acetaminofén. 
Enero - abril

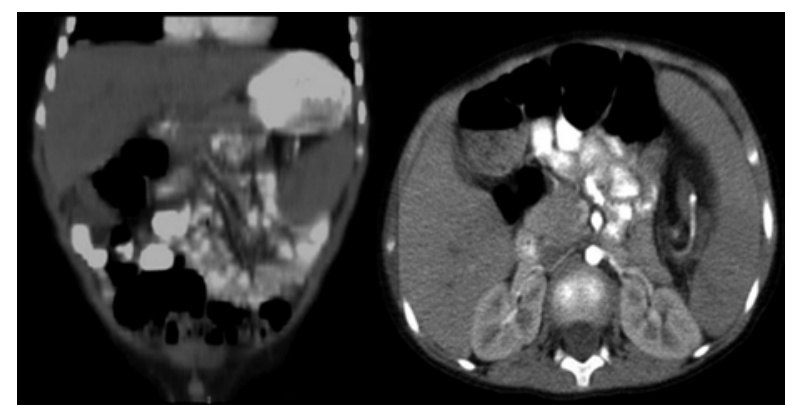

Figura 1: TC de abdomen contrastado realizad a los 3 años de vida, con evidencia de bazo con implantación baja y adecuada perfusión esplénica.

Fuente: autores

Al ingreso, se encontró con deshidratación grado I, con taquicardia, febril y sensación de masa a nivel de fosa ilíaca derecha, dolorosa a la palpación y sin signos de irritación peritoneal. Se consideró inicialmenteque cursaba con probable plastrón apendicular, por lo que se inició manejo antibiótico (ampicilina/ sulbactam), líquidos endovenosos y reposo intestinal. Los paraclínicos de ingreso mostraron leucocitosis $21.400 \mathrm{cel} / \mathrm{mm}^{3}$ con neutrofilia y proteína $C$ reactiva negativa. Se realizó TACc con hallazgo sugestivo de bazo ectópico, torsión del pedículo vascular y compromiso isquémico (Ver Figura 2), razón por la cual se decidió llevar a laparoscopia exploratoria de urgencia.

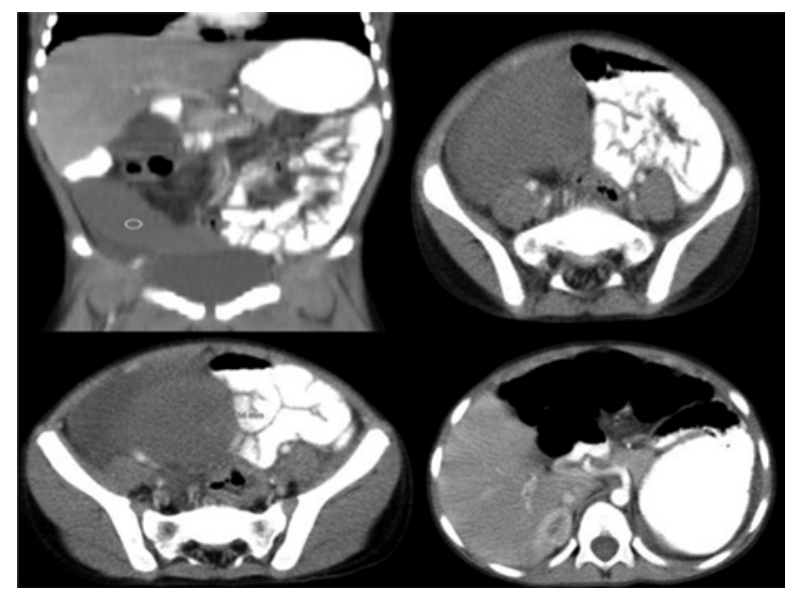

Figura 2 TACc abdominal: bazo ectópico a nivel de fosa ilíaca derecha, con líquido periesplénico.

Fuente: autores

En primer tiempo laparoscópico, se evidenció división del ligamento gastroesplénico por el antecedente
Torsión esplénica con localización inusual, reporte de caso quirúrgico descrito, bazo ectópico en fosa iliaca derecha con proceso inflamatorio perivisceral y el pedículo vascular esplénico rotado en $720^{\circ}$ (Ver Figura 3.A). Se realizó detorsión del pedículo vascular y ante la no claridad de la relación del mismo con el meso del colon, se realizó laparotomía exploratoria, logrando recuperación del flujo vascular del bazo, por lo que se realizó conservación esplénica con esplenopexia a nivel de hipocondrio izquierdo, utilizando 4 puntos separados de Prolene vascular 3-0 entre el diafragma y el peritoneo parietal lateral de la pared abdominal, y rotando un colgajo pediculado de omento mayor para evitar efecto de cizallamiento esplénico con el material de sutura.(Ver Figura 3.C)



Figura 3.A: Hallazgo intraoperatorio. Bazo en ubicación ectópica en fosa ilíaca derecha, con pedículo laxo, torsión del mismo y con signos de isquemia.

Fuente: autores

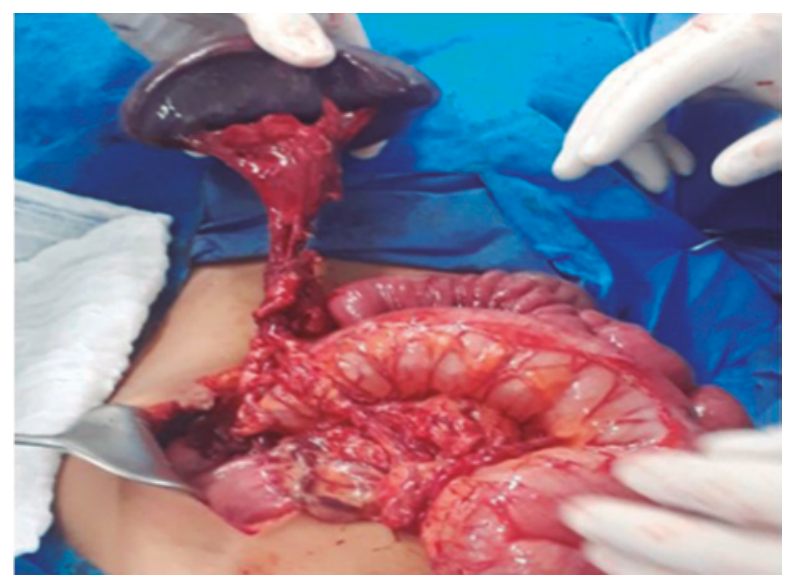

Figura 3.B: Hallazgo intraoperatorio. Bazo posterior a la detorsión del pedículo vascular, con mejoría de la perfusión del órgano. Fuente: autores 


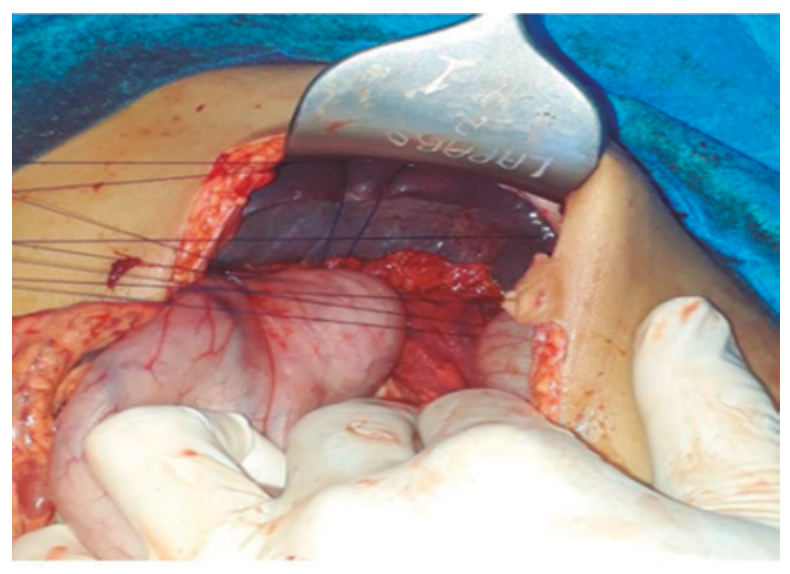

Figura 3.C: Esplenopexia con suturas de prolene vascular 3-0, entre peritoneo parietal y diafragma.

Fuente: autores

El paciente presentó evolución posquirúrgica inmediata adecuada, con Doppler esplénico a las 24 horas que evidenció bazo bajo la modalidad doppler, pulsado y codificado por color curvas espectrales fasicas normales y adecuadas en el hilio esplénico, confirmando adecuada perfusión esplénica. Se realizó manejo de dolor posquirúrgico con catéter peridural con infusión de bupivacaína al $0.1 \%$, que fue retirado al tercer día posquirúrgico. Presentó íleo posquirúrgico en el cuarto día (Ver Figura 4) se dio manejo médico (reposo gástrico, sonda nasogástrica, deambulación e hidratación endovenosa) con evolución clínica satisfactoria a las 48 horas, por lo que se indicó egreso.

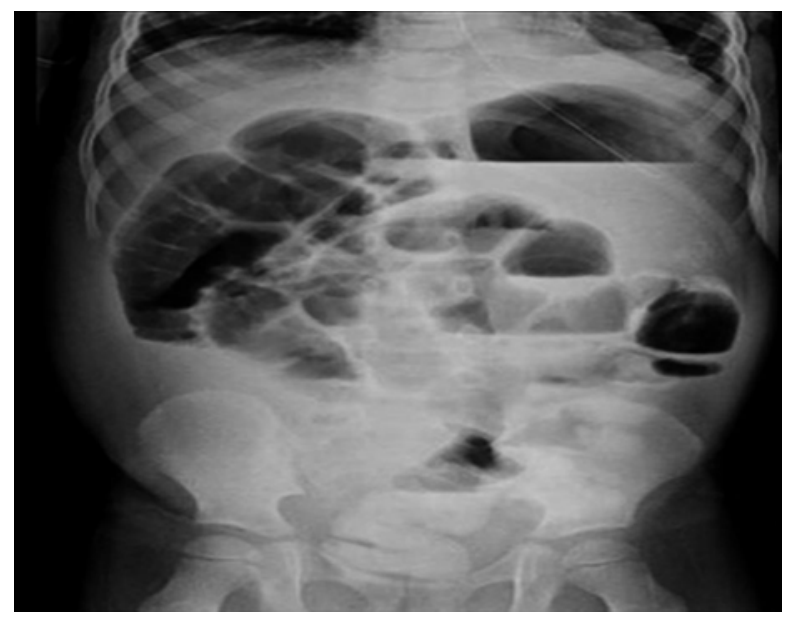

Figura 4. Radiografía de abdomen decúbito supino con hallazgos sugestivos de íleo posquirúrgico.

Fuente: autores
Fue revalorado de manera ambulatoria 30 días posterior a su egreso, confirmando buen estado clínico y adecuada función esplénica dada por normalización progresiva en parámetros hematológicos, hallando disminución del recuento plaquetario evidenciado en $700.000 \mathrm{cel} / \mathrm{mm}^{3}$, que durante el postoperatorio alcanzó valores hasta de $1.615 .000 \mathrm{cel} / \mathrm{mm}^{3}$, se encontró normalización de hemoglobina $12 \mathrm{~g} / \mathrm{dl}$, previamente reportada en $9.4 \mathrm{~g} /$ dl, sin requerir medidas de intervención adicionales.

\section{Discusión}

El bazo errante se ha descrito como una entidad poco frecuente, que suele presentarse en la infancia y en adultos entre los 20 y 40 años, especialmente en mujeres con respecto a hombres, en una proporción de $20: 1^{2}$.

En algunos reportes de la literatura de esta entidad, no se encuentran antecedentes quirúrgicos claros (500 casos reportados desde 2002) $)^{11}$, existe poca evidencia de pacientes con antecedente de reparación de hernia diafragmática congénita como en el caso descrito por Metha et $a^{3}$ y/o Funduplicatura de Nissen en el reporte de Khoi et $a^{11}$. En este procedimiento se incluye en la técnica quirúrgica la ligadura del ligamento gastroesplénico y de los vasos gástricos cortos con el objetivo de disminuir la morbilidad posquirúrgica y la tensión de la funduplicatura, sin embargo puede facilitar la movilidad esplénica, como en el caso clínico reportado.

El 70\% al $80 \%$ de los casos reportados son mujeres en edad fértil ${ }^{12}$ y en un tercio de los casos se encuentra en niños, con prevalencia de niñas respecto a niños en mayores de 1 año 4 .

La forma congénita de la rotación esplénica se presenta debido a una laxitud o ausencia de los ligamentos suspensorios del bazo (esplenorenales, gastroesplénicos, esplenocólicos y esplenoperitoneal), lo que predispone a una mayor movilidad del órgano dentro de la cavidad abdominal y posible torsión a lo largo del pedículo vascular'3.

\section{Diagnóstico:}

Ante la baja frecuencia descrita, la sospecha clínica, (dolor abdominal crónico, la sensación de masa abdominal y en algunos casos alteraciones de las 


\section{Enero - abril}

líneas hematológicas y/o antecedentes quirúrgicos ya descritos) y el conocimiento de la entidad es importante para lograr un diagnóstico oportuno y enfoque adecuado ${ }^{11-14}$.

Dentro de las manifestaciones clínicas del paciente del presente caso, se resalta la importancia del dolor abdominal crónico, intermitente, con agudización del cuadro, dada por aumento del dolor y síndrome febril asociado, posiblemente secundario a la torsión del pedículo vascular esplénico, que son signos y síntomas similares a los encontrados en los diferentes casos reportados ${ }^{12-15}$ Adicionalmente, se pueden encontrar alteraciones inespecíficas en las pruebas de laboratorio, como leucocitosis y en algunos casos trombocitosis o trombocitopenia asociada ${ }^{2-16}$.

De acuerdo a la presentación, los estudios de imagen tienen gran utilidad como herramienta diagnóstica. En pacientes con casos de dolor abdominal crónico e intermitente, la ecografía abdominal total + Doppler permite advertir la localización anormal del bazo y determinar alteraciones en la perfusión del mismo, y en los casos agudos, la TACc, tiene mayor rendimiento, ya que permite diagnosticar la entidad al tiempo que descarta otras causas de dolor abdominal 7 .

A pesar del antecedente de $\mathrm{FN}$ en nuestro paciente, la impresión diagnóstica inicial fue plastrón apendicular, razón por la que se solicitó TACc (Ver Figura 2), en el que se logró el diagnóstico de bazo ectópico con torsión del pedículo y se llevó a cirugía de urgencia.

\section{Tratamiento:}

El tratamiento del bazo errante es quirúrgico. Se reconoce que la mortalidad intraoperatoria es inferior al $1 \%$, pero se han descrito complicaciones hasta del $65 \%$ de los casos no tratados quirúrgicamente ${ }^{2}$. Sin embargo, debido al reconocimiento de la importante función inmunológica del bazo y a la alta incidencia de sepsis postesplenectomía, especialmente en la población pediátrica, se prefiere la conservación esplénica con esplenopexia siempre que sea posible, es decir, en ausencia de hallazgos de infarto esplénico masivo o trombosis venosa esplénica que obligarían a realizar una esplenectomía 3-12.

Se describe en la literatura la realización de detorsión del pedículo esplénico vía abierta o laparoscópica
Torsión esplénica con localización inusual, reporte de caso

asociado a esplenopexia, en el cual se utilizan materiales protésicos tipo malla de polipropileno, prótesis de material absorbible, o incluso la creación de bolsillo peritoneal para lograr la fijación adecuada del bazo en su localización habitual ${ }^{14}$. En el presente caso, se realizó detorsión del pedículo esplénico comprobando reperfusión exitosa y se llevó a cabo la esplenopexia descrita debido a la edad del paciente, adicionalmente, el moderado proceso inflamatorio disminuía la posibilidad de creación de bolsillo peritoneal y, con el fin de reducir al máximo la probabilidad de adherencias peritoneales vs fístula asociada a materiales protésicos se consideró como mejor opción la realización de esplenopexia con la técnica descrita, obteniendo resultados posteriores satisfactorios.

\section{Conclusiones}

La rotación esplénica es una patología de baja frecuencia. Su diagnóstico depende del conocimiento previo de la entidad y de la identificación de factores de riesgo en el paciente. La Tomografía computarizada con contraste es la técnica de imagen de elección y, posterior al diagnóstico, se debe ofrecer un tratamiento oportuno que permita tanto la conservación del bazo como su respectiva fijación, esto con el fin de evitar esplenectomía, siempre que sea posible, y la morbimortalidad asociada.

\section{Referencias bibliográficas}

1. Misawa T, Ph D, Yoshida K, Shiba H, Kobayashi S, Kanaga K. Wandering spleen with chronic torsion. Am J Surg. 2008;195(4):504-5.

2. Maestre Serrano YR, Acevedo-reyes H, Cohen-Ríos S, Álvarezjiménez J. Bazo errante gigante con torsión del pedículo e infarto esplénico : Reporte de un caso. Rev Cir. 2019;71(3):25760.

3. Mehta A, Vana PG, Glynn L. Splenic torsion after congenital diaphragmatic hernia repair: Case report and review of the literature. J Pediatr Surg. 2013;48(3):29-31.

4. Brown BCVR, Virgilio GR, Vazquez WD. Wandering spleen and its complications in children: a case series and review of the literature. J Pediatr Surg. 2003;38(11):1676-9.

5. Anand S, Yadav DK, Sharma S, Varshney A. Acute torsion of a wandering spleen: a paediatric emergency. BMJ Case Rep. 2018:2-3.

6. Cohen O, Baazov A, Samuk I, Schwarz M, Kravarusic D, Freud E. Emergencies in the Treatment of Wandering Spleen. Isr Med Assoc J. 2018;20(6):354-7.

7. Khan DB, Khandwala K, Abbasi S, Khan SD, Raza R. Torsion of Wandering Spleen with Infarction. Cureus. 2018;10(8):8-12.

8. Nemcek A, Miller FH, Fitzgerald SW. Acute Torsion of a Wandering Spleen: Diagnosis by CT and Duplex Doppler and Color Flow Sonography. AJR Am J Roentgenol. 1991;157:307-9.

9. Ghahremani S, Sabour M, Ghahremani S, Bazrafshan A, Sadeghi R. A wandering spleen detected by $99 \mathrm{~m}$ Tc-denatured RBC scan. Iran J Nucl Med. 2018;26(1):66-7. 
10. Swischuk LE, Williams JB, John SD. Torsion of wandering spleen : the whorled appearance of the splenic pedicle on CT. Pediatr Radiol. 1993;23:476-7.

11. Le K, Griner D, Hope WW, Tackett D. Splenic Torsion Requiring Splenectomy Six Years Following Laparoscopic Nissen Fundoplication. JSLS. 2012;16(1):184-8.

12. Steinberg BR, Karmazyn B, Dlugy E, Gelber E, Freud E, Horev G, et al. Clinical Presentation of Wandering Spleen. J Pediatr Surg. 2002;37(10):1-4.

13. Palanivelu C, Rangarajan M, Senthilkumar R, Parthasarathi
R, Kavalakat AJ. Laparoscopic Mesh Splenopexy (Sandwich Technique) for Wandering Spleen. JSLS. 2007; 11(2):246-51.

14. Vásquez JL, Montero M, Díaz F, Muguerza R, Páramo C, Rodríguez-Costa A. Acute torsion of the spleen: diagnosis and management. Pediatr Surg Int. 2004;20(2):153-4.

15. Chu J, Li Z, Luo B, Yang J. Wandering spleen with torsion and complete infarction. Acta Radiol. 2011;52(8):911-3.

16. Cecchetto G, Dall'Igna P, Nogare CD, Gasparella P, Zanon GF. Acute splenic torsion in children: which is the best treatment? A case report. Pediatr Med Chir. 2012;34(3):146-7. 